\title{
Effect of Temperature on Anisotropic Crystal Growth of Boehmite under Hydrothermal Condition
}

\author{
Tatsuya Fujii, Shin-ichiro Kawasaki and Mitsuhiro Kanakubo \\ Research Institute for Chemical Process Technology, National Institute of Advanced Industrial Science and Technology, 4-2-1 \\ Nigatake, Miyagino-ku, Sendai, Miyagi 983 8551, Japan
}

\begin{abstract}
Temperature effect $\left(200-400{ }^{\circ} \mathrm{C}\right)$ on the anisotropic crystal growth of boehmite under hydrothermal conditions with and without octanoic acid was investigated. The crystallinity and the size of particles increased with increasing the treatment temperature. The crystal growth was facilitated greatly above the critical temperature of water. Although several possible factors could affect the crystal growth behavior, the experimental results were discussed in terms of water properties, such as dielectric constant and viscosity. The crystallization was enhanced when the viscosity and dielectric constant of water were low. The viscosity reduction at higher temperature may enhance diffusion and crystallization, in particular, without octanoic acid. The enhancement of crystallization at lower dielectric constant implies that the formation of particles, which are less polar than precursor ions, favorably proceeds in such media. The crystal growth along c-axis showed less temperature dependence around the critical temperature in the experiments with octanoic acid, which suggests that the modification reaction on the (001) surface was also facilitated because the modification reaction forms less polar products. This is probably the reason why the aspect ratio $(\mathrm{a} / \mathrm{c})$ was considerably higher for the products obtained with the treatments above the critical temperature.
\end{abstract}

Key words: Crystal morphology, hydrothermal crystal growth, inorganic compounds, nanomaterials.

\section{Introduction}

Demand for high-performance materials has been increasing. Organic films are widely used as such materials. To improve the performance of organic films, the addition of inorganic filler particles is one of the promising approaches. In general, however, the affinity of inorganic particles to organic polymers is poor, which leads to aggregation and contact failure of particles with organic polymers[1, 2]. To achieve better performance of polymer composites, the affinity of inorganic particles to organic polymers is important.

For improving particle affinity to organics, surface modification is one of the promising candidates. For example, several researchers have reported that silane coupling treatments of particles enhance affinity to organics [3]. Another method was proposed by

Corresponding author: Tatsuya Fujii, Ph.D., research field: chemical engineering.
Adschiri et al. for in situ organic surface modifications under supercritical hydrothermal condition [4]. In this technique, an aqueous metal salt solution with an organic modifier, e.g. carboxylic acid, is used as the starting material, and the hydrothermal synthesis proceeds under supercritical hydrothermal condition. Since the organic modifier coexists in the crystal formation field, the modification reaction takes place effectively, which produces the nano-sized particles capped with the organic modifier. The high temperature treatment can successfully provide the nanoparticles with high crystallinity.

In addition to the affinity to organics, the shape of filler particles is important to obtain desired performance of polymer composites. In general, shielding performance can be raised effectively by plate-like particles [5], while conductivity and mechanical strength can be upgraded efficiently by particles with high aspect ratios [6-8]. As one of methods for controlling particle shape, the 
hydrothermal crystallization using capping agent has also been proposed [9]. It has been reported that the particle shape synthesized under supercritical hydrothermal condition with a carboxylic acid was controlled by the capping effect [10], although the size and aspect ratio of particles did not change drastically because the reaction was terminated immediately after nucleation.

The authors have applied the organic modification syntheses under supercritical hydrothermal condition to the crystal growth step of a particle, boehmite ( $\mathrm{AlOOH}$ or $\mathrm{Al}_{2} \mathrm{O}_{3} \cdot \mathrm{H}_{2} \mathrm{O}$ ), aiming the high aspect ratio particle with high affinity to organics [11]. A boehmite can be used as a filler to raise mechanical strength and thermal conductivity of polymers [7, 12]. The authors used a boehmite powder with low crystallinity as a starting material, and treated it under supercritical hydrothermal condition with a variety of carboxylic acids. The authors found that the shape of boehmite was varied to the long hexagonal plate from the rhombic plate by adding some carboxylic acids in supercritical water, and the anisotropic crystal growth proceeded rapidly [11]. It has been suggested that the particle shape was controlled by the capping effect and the crystal growth was facilitated by the existence of carboxylic acids.

In the above crystal growth process, there should exist several elementary steps. Assuming that the crystal growth occurred with the dissolution-recrystallization process as suggested by the previous study [11], one has to take account of dissolution of powder into precursors such as $\mathrm{Al}^{3+}$, $\mathrm{Al}(\mathrm{OH})^{2+}, \quad \mathrm{Al}(\mathrm{OH})_{2}{ }^{+}, \quad \mathrm{Al}(\mathrm{OH})_{3}$ and $\mathrm{Al}(\mathrm{OH})_{4}^{-}$, transportation in solution, diffusion on particle surface, crystallization reaction, and modification reaction. The previous studies also imply the existence of intermediates consisting of particle precursors and modifiers, which can enhance the crystal growth of particles $[10,11,13]$. These steps have their own temperature dependences, and at certain temperature the specific step(s) should play the rate-determining role. Hence, the treatment temperature should be appropriately optimized to obtain desired particles.

In addition to such temperature effects, the properties of water such as dielectric constant [14] and viscosity [15] are of great importance because they significantly vary with temperature especially near the critical point of water $\left(374{ }^{\circ} \mathrm{C}, 22.1 \mathrm{MPa}\right)$. It has been pointed out that the changes in properties of water by temperature and pressure can drastically affect some reaction rates in sub- and supercritical water. For example, the hydrolysis of $\mathrm{CH}_{2} \mathrm{Cl}_{2}$ was diminished under supercritical conditions by the reduction of dielectric constant as $\mathrm{CH}_{2} \mathrm{Cl}_{2}$ is less polar and more stable than the products in lower dielectric constant media [16]. In crystallization processes, analogically, if the polarities of precursors are higher than those of the products, the crystallization could be facilitated under lower dielectric constant conditions. This was suggested by the previous study for the nanoparticle syntheses such as $\mathrm{CeO}_{2}$ and $\mathrm{NiO}$ under supercritical water without modifiers [17].

The authors have previously studied the crystal growth behavior of boehmite under supercritical hydrothermal condition at $400{ }^{\circ} \mathrm{C}$ [11]. In this study, the temperature range examined was extended (between 200 and $400{ }^{\circ} \mathrm{C}$ ), and the effect of temperature on the anisotropic crystal growth of boehmite with octanoic acid was investigated. Although several temperature effects should be considered to optimize the present complicated process, the authors attempt to understand the temperature effect by change in water properties such as dielectric constant and viscosity.

\section{Experimental}

Hydrothermal treatment of boehmite powder (Pural SCF, Sasol Ltd.) was conducted using a batch type reactor made of SUS316 stainless steel. The inner volume of the reactor was $8.5 \mathrm{~cm}^{3}$. The experimental procedure was similar to the previous research [11]. The temperature effect was investigated from 200 to 
$400{ }^{\circ} \mathrm{C}$. A $3.0 \mathrm{~g}$ of water and $0.15 \mathrm{~g}$ of boehmite was added in the reactor for the experiments below the critical temperature ca. $374{ }^{\circ} \mathrm{C}$, while the amount of water added in the reactor was determined so that the pressure of water to be $30 \mathrm{MPa}$ for the experiments above the critical temperature. As a modifier, octanoic acid (Wako Chemicals) was used. The octanoic acid was added in the reactor; the concentration at ambient condition was fixed to be $3.2 \mathrm{~mol} / \mathrm{L}$.

XRD (X-ray diffraction) analyses were conducted to determine the crystal phase of product particles and to evaluate the crystallinity using an Intelligent XRD system (SmartLab, Rigaku) equipped with a $\mathrm{Cu} \mathrm{K} \alpha$ radiation source. The amounts of water and modifier in particles attached to the surface of products were evaluated using a thermogravimetric analyse (TGA, TG-DTA2000, Bruker Corp). The particle shape of products was observed using a transmission electron microscope (TEM, TecnaiG2 30, FEI Co.). The analytical procedure was the same as the previous study, which was described elsewhere [11].

\section{Results and Discussion}

\subsection{Characterization of Products Obtained after 10} min Treatments at Different Temperatures

The authors conducted $10 \mathrm{~min}$ hydrothermal treatments of boehmite with and without octanoic acid.
The particle shapes were observed using a TEM. Fig. 1 compares the TEM images of products obtained after the hydrothermal treatments without octanoic acid at different temperatures. The particle images are not clear for the products treated at $200{ }^{\circ} \mathrm{C}$; the particle shape seems to be rounded-rhombic plate. The particle images are more clearly observed for the products treated at the temperatures of $250-400{ }^{\circ} \mathrm{C}$; the particle shape is rhombic plate. As the treatment temperature increases, the size of particle tends to become larger.

Fig. 2 shows the TEM images of products obtained after the hydrothermal treatments with octanoic acid at different temperatures. As the TEM images obtained without octanoic acid, the particle images are not clear for the products treated at $200{ }^{\circ} \mathrm{C}$; the particle shape looks like elliptical plate. When the treatment temperature increases, the particle images become clearer and the shapes turn to hexagonal plate with higher aspect ratios. The aspect ratio tends to increase with increasing the treatment temperature.

The crystal phases of the products obtained after the hydrothermal treatments were analyzed by XRD in Fig. 3. All the products were confirmed to be orthorhombic boehmites (JCPDS 74-1895), as demonstrated for the particles treated at $400{ }^{\circ} \mathrm{C}$ in the previous study [11]. Irrespective of the presence of
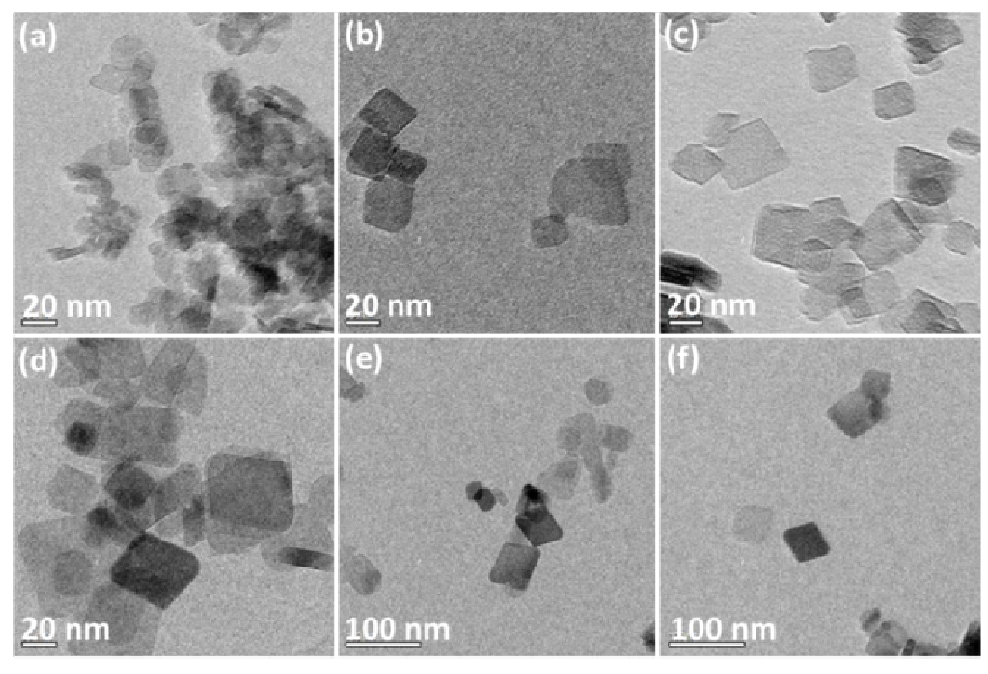

Fig. 1 TEM images obtained after hydrothermal treatments without octanoic acid at different temperatures of (a) $200{ }^{\circ} \mathrm{C}$; (b) $250{ }^{\circ} \mathrm{C}$; (c) $300{ }^{\circ} \mathrm{C}$; (d) $350{ }^{\circ} \mathrm{C}$; (e) $380{ }^{\circ} \mathrm{C}$; (f) $400{ }^{\circ} \mathrm{C}$. 


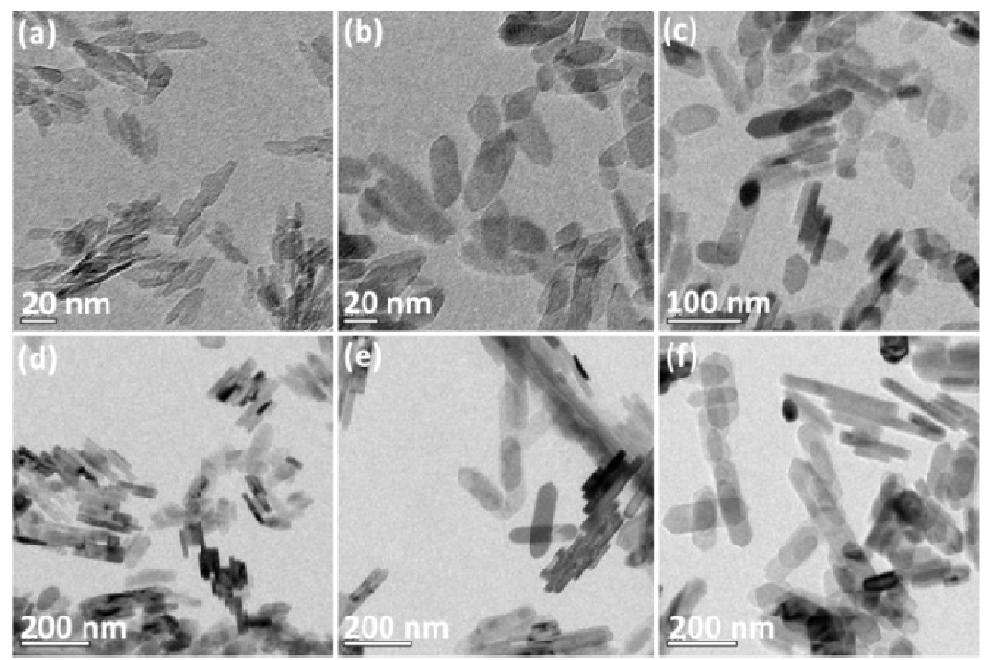

Fig. 2 TEM images obtained after hydrothermal treatments with octanoic acid at different temperatures of (a) $200{ }^{\circ} \mathrm{C}$; (b) $250{ }^{\circ} \mathrm{C}$; (c) $300{ }^{\circ} \mathrm{C}$; (d) $350{ }^{\circ} \mathrm{C}$; (e) $380{ }^{\circ} \mathrm{C}$; (f) $400{ }^{\circ} \mathrm{C}$.
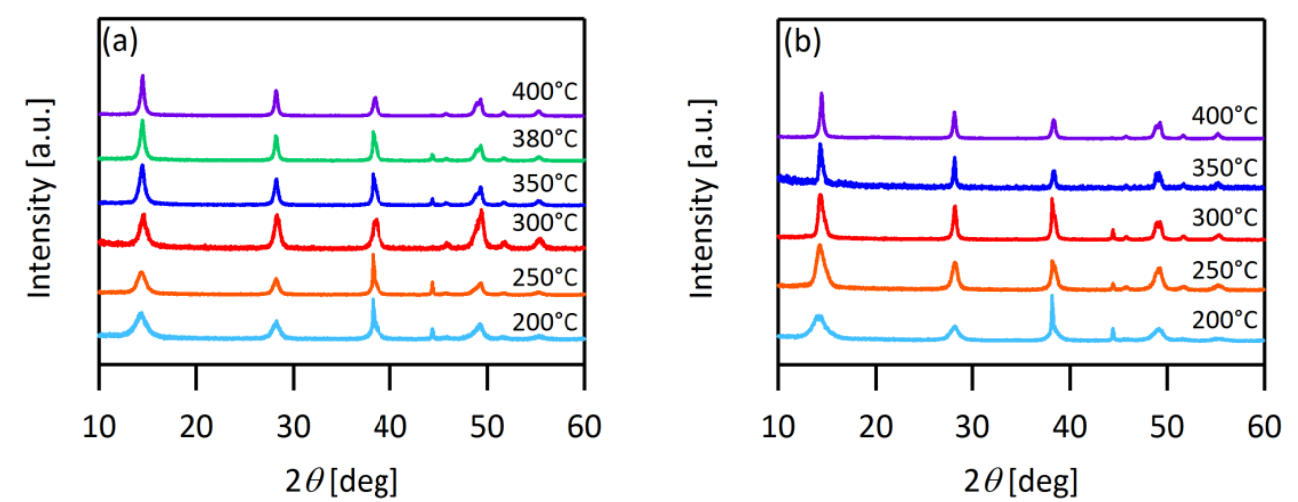

Fig. 3 Comparison of XRD spectra for particles obtained after treatments (a) without additives; and (b) with octanoic acid at different temperatures.

octanoic acid, the peaks of XRD spectra become sharper with increasing temperature in the hydrothermal treatments. This suggests that the residual water molecules in the orthorhombic boehmites were reduced and the crystallinity was increased with increasing temperature.

To evaluate the thermal stability of products and confirm the existence of octanoic acid on the products, the authors conducted TGA for the products in Fig. 4. The TG losses beyond $130{ }^{\circ} \mathrm{C}$ were evaluated to exclude the effect of water physically adsorbed on the surface. Note that the large TG loss up to approximately $15 \%$ observed around $450{ }^{\circ} \mathrm{C}$ derives from dehydration from boehmite particles [11]. After the dehydration, -alumina $\left(\mathrm{Al}_{2} \mathrm{O}_{3}\right)$ is obtained $\left(2 \mathrm{AlOOH} \rightarrow \mathrm{Al}_{2} \mathrm{O}_{3}+\right.$ $\mathrm{H}_{2} \mathrm{O}$ ). The results for the particles without additives in
Fig. 4a indicate that the TG loss started at lower temperature for the products treated at lower temperature. At $800^{\circ} \mathrm{C}$, the particles treated at lower temperature reached the lower TG values. The TG loss at low temperatures around $150-250{ }^{\circ} \mathrm{C}$ is probably due to the dehydration of the surface $\mathrm{OH}$ group of boehmite. Since the particles treated at lower temperatures are smaller and those surface areas are relatively greater, the TG loss by the dehydration of the surface $\mathrm{OH}$ group should be larger for the products treated at lower temperatures. In addition, the amount of water captured in boehmite is considered to be larger for the products treated under lower temperatures, in view of the lower crystallinity indicated by the XRD patterns. The TG loss for octanoic acid modified products in Fig. 4b should include the contributions 

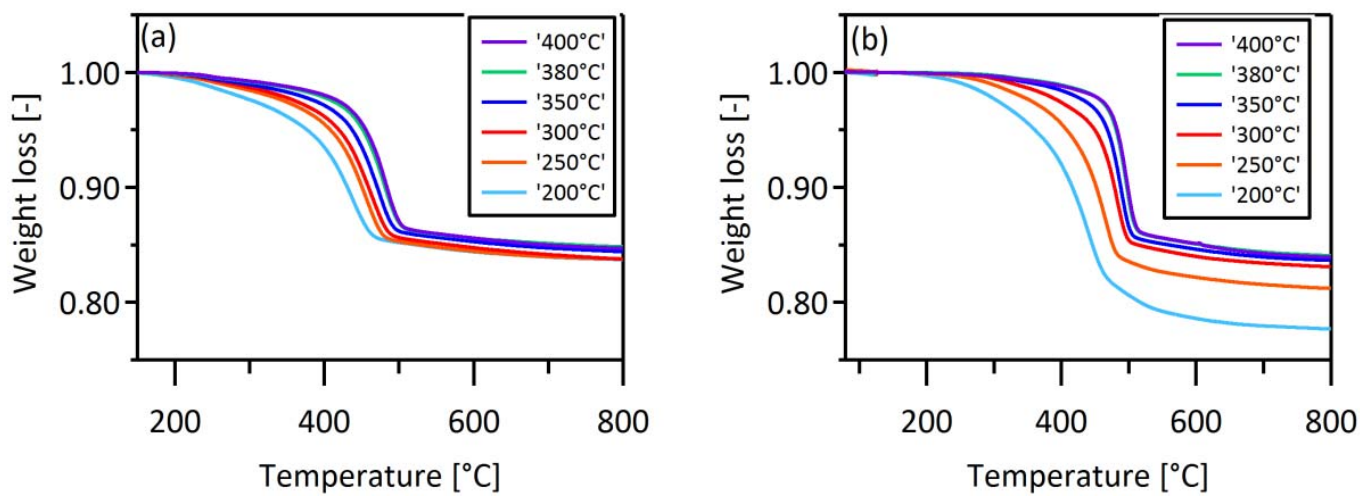

Fig. 4 Comparison of TG curves of particles obtained after treatments (a) without additives and (b) with octanoic acid at different temperatures.

from not only dehydration but also octanoic acid elimination. A comparison of Figs. $4 \mathrm{a}$ and $4 \mathrm{~b}$ indicates that the TG losses for the products treated with octanoic acid were larger than those without additives at each temperature. Thus, the surface modification by octanoic acid proceeds effectively at all the temperatures performed in this study.

From the above results, it has been concluded that more crystalline particles with defined shapes can be obtained after the treatments at higher temperatures. In addition, the size of particle becomes greater at higher temperatures. In the next section, the temperature effects on the lengths of particles are discussed in detail.

\subsection{Temperature Effects on Lengths of Particles}

The authors evaluated the lengths of the particles obtained after the treatments at different temperatures. A method for determining the particle lengths and the definition of a-, b- and c-axis are the same as the previous research [11]. Briefly, a-axis corresponds to the long-axis, b-axis to the thick-axis, and c-axis to the short-axis of the particles. In this study, to discuss the change in size and shape of particles, the normalized lengths were employed, where the length of each axis was divided by each mean length of the product obtained after 10 min treatment without octanoic acid at $400{ }^{\circ} \mathrm{C}$ (a-axis $=68.7 \mathrm{~nm}$, b-axis $=18.1 \mathrm{~nm}$, and c-axis $=75.2 \mathrm{~nm})$.
Fig. 5 shows the temperature effects on normalized lengths (a) without and (b) with octanoic acid. The results for the particles without additives in Fig. 5a indicate that the normalized lengths increased up to unity with increasing temperature, and the temperature effect on the normalized length of each axis was nearly the same. Although the temperature effect is small at subcritical temperatures $\left(200-350{ }^{\circ} \mathrm{C}\right)$, the normalized lengths jumped up beyond the critical temperature $\left(\sim 374^{\circ} \mathrm{C}\right)$. The apparent activation energy between $250-350{ }^{\circ} \mathrm{C}$ is very small $(6-9 \mathrm{~kJ} / \mathrm{mol})$, which suggests that the diffusion of ionic species in the bulk solution may be the rate-determining step[18]. On the other hand the activation energy between $380-400{ }^{\circ} \mathrm{C}$ is much higher $(29-30 \mathrm{~kJ} / \mathrm{mol})$. The rate-determining step may be altered, as one of the reasons for the observed temperature effect. In addition to the above possibility, the changes in water properties may affect the temperature tendency. The viscosity of water steeply decreases around the critical temperature, so the diffusion should be enhanced greatly when the treatment temperature is raised over the critical temperature. Moreover, the dielectric constant of water also decreases steeply. The decrease in dielectric constant should stabilize non-polar species, which can enhance the crystallization because the product crystals are considered to have less polarity than the precursor ions. Such effects may be integrated in the values of apparent activation energies. 
Fig. $5 \mathrm{~b}$ indicates that the normalized lengths for the particles with octanoic acid increased beyond unity with increasing temperature, and the temperature effect on normalized lengths were dependent on the axes. The most remarkable increase was observed for the length along a-axis, followed by $b$ - and c-axes. The activation energies even in the lower temperature range between $200-350{ }^{\circ} \mathrm{C}$ were greater than 20 $\mathrm{kJ} / \mathrm{mol}(28-30 \mathrm{~kJ} / \mathrm{mol})$. Hence, the rate-determining step is considered to be some more complex processes, involving the modification reactions on the surface of particles, such as the crystallization, solvation/desolvation, diffusion, and so on. It is considered that the presence of octanoic acid alters the rate-determining step. It is also found that the temperature effect becomes greater at higher temperatures $\left(350-400{ }^{\circ} \mathrm{C}\right)$ for the lengths along aand $\mathrm{b}$-axes, whereas the length along c-axis has less temperature dependence over the temperature investigated. The possible explanation is as follows. The crystallization with octanoic acid can be promoted at higher temperatures as similar to the case without octanoic acid, but the surface modification reactions may form the less polar product species and the reactions can be facilitated in the lower dielectric constant media. Because the capping effect is larger on the (001) surface as demonstrated in the previous work [11], the crystal growth enhancement for the c-axis at higher temperature was considered to be cancelled.

Fig. 6 presents the aspect ratio (the length along a-axis divided by that along c-axis) of products obtained after the treatments with octanoic acid at different temperatures. It has been confirmed that the aspect ratio increased steeply with temperature around $350-400{ }^{\circ} \mathrm{C}$ because of the rapid crystal growth of particles along a-axis against c-axis.

To further investigate the effect of temperature on the crystal growth behavior of boehmite particles, the temporal variations of normalized lengths were investigated at the different temperatures of 300,350 and $400{ }^{\circ} \mathrm{C}$. The data at $400{ }^{\circ} \mathrm{C}$ were cited from the previous work [11]. Fig. 7 compares the temporal variations of normalized lengths. At $400{ }^{\circ} \mathrm{C}$, the normalized lengths increased with increasing the treatment time until 30-60 min for all the axes. At 300 and $350{ }^{\circ} \mathrm{C}$, on the other hand, the normalized lengths stopped growing around $10 \mathrm{~min}$. This implies that when the modification proceeds enough, the crystal growth stops in the treatments at lower temperatures. The capping effect at higher temperatures may be lower because of much greater diffusivity under supercritical conditions, which can favorably result in the higher aspect ratio products.

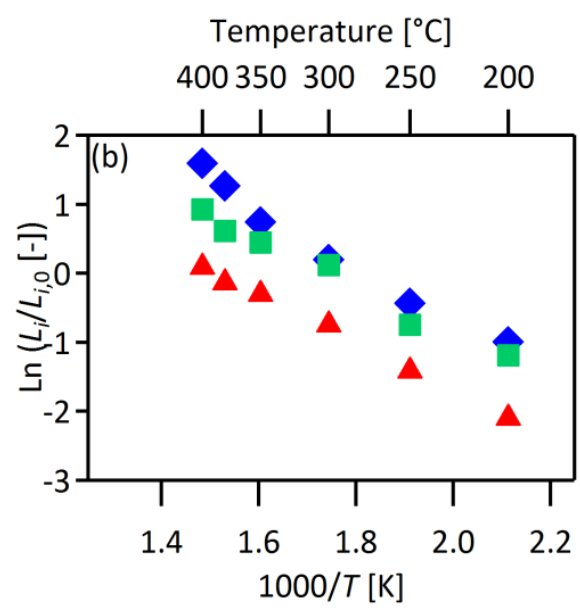

Fig. 5 Comparison of normalized lengths of particles obtained after treatments (a) without additives and (b) with octanoic acid at different temperatures (diamond: a-; square: b-; triangle: c- axis). 


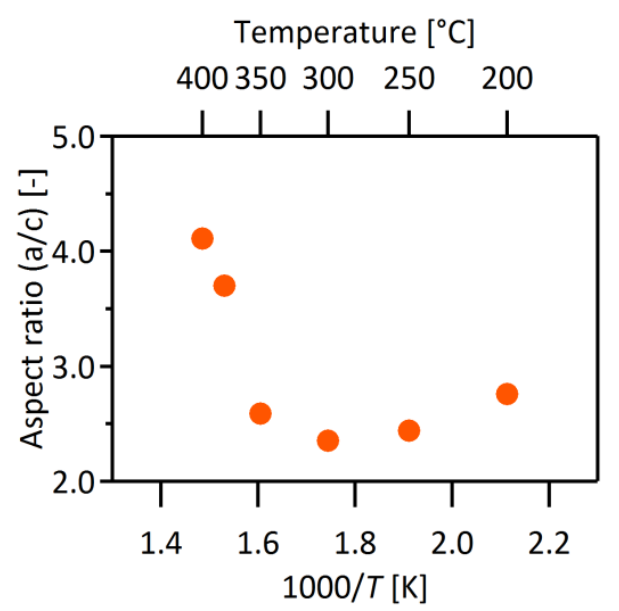

Fig. 6 Effect of temperature on aspect ratio (a/c) of particles obtained after treatments with octanoic acid.
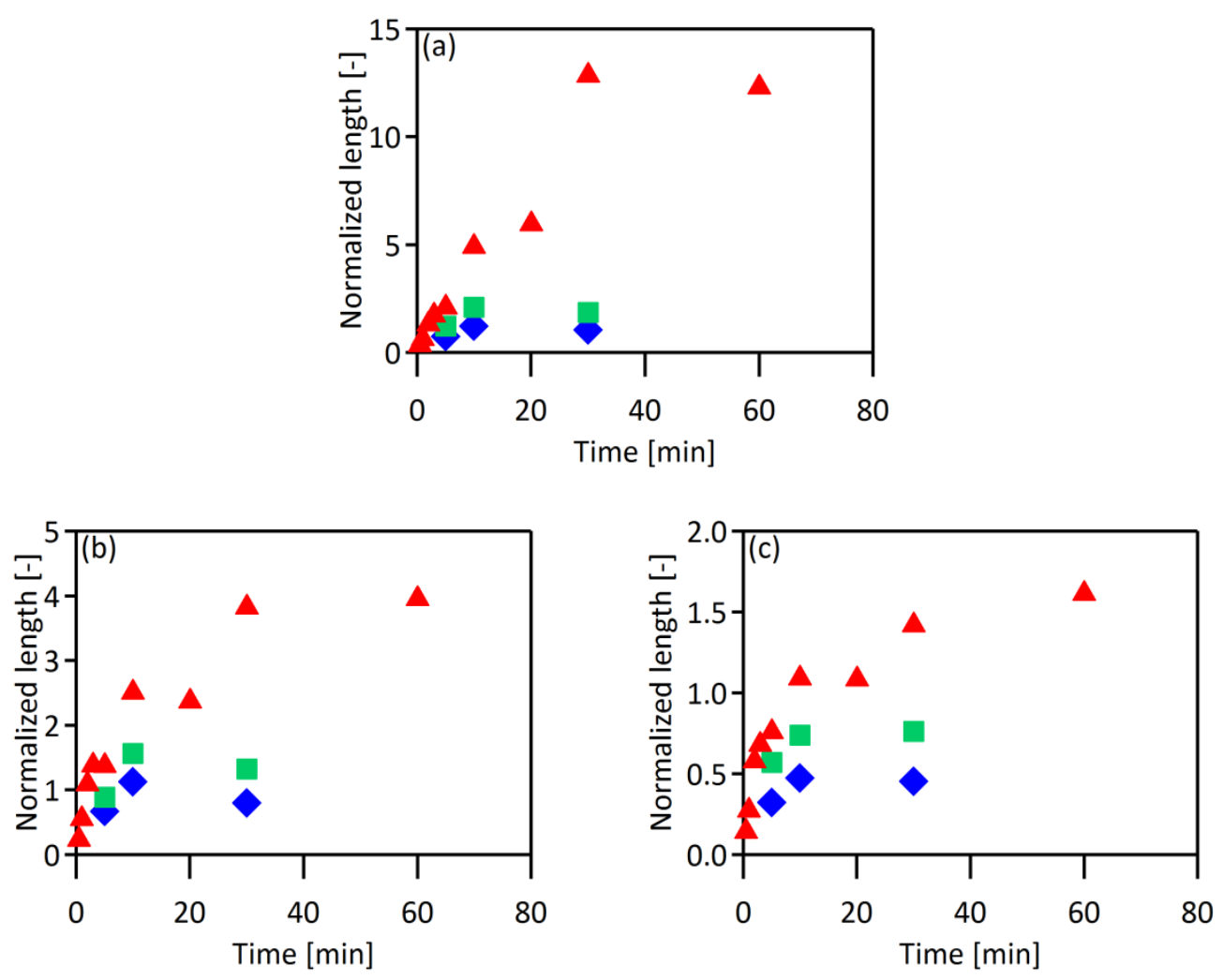

Fig. 7 Comparison of temperature effect on temporal variations of normalized lengths along (a) a-axis, (b) b-axis, and (c) c-axis (diamond: 300 ; square: 350 ; triangle: $400{ }^{\circ} \mathrm{C}$ ).

\section{Conclusions}

The temperature effect on the anisotropic crystal growth of boehmite under hydrothermal conditions with and without octanoic acid was experimentally investigated. The crystallinity and the size of particles increased with increasing the treatment temperature. The change in water properties such as viscosity and dielectric constant is considered to be one of the predominant factors that enhance the crystal growth above the critical temperature. The crystal growth along c-axis was not effective, which resulted in the 
higher aspect ratios $(\mathrm{a} / \mathrm{c})$ of products at higher temperature above the critical point. There are several possible factors that could affect the crystal growth behavior such as solubility of boehmite, intermediate formation, diffusion rate, and so on. The contributions of these factors have not yet been clarified quantitatively in this study, and further investigation will be carried out in due course to optimize the hydrothermal process.

\section{Acknowledgement}

The authors thank Sasol Ltd. for donating a boehmite powder (Pural SCF). The authors also thank Ms. Ryoko Hiraoka and Mr. Ken-ichro Otaki for their assistance with the experiments and analyses.

\section{References}

[1] Deng, H., Lin, L., Ji, M., Zhang, S., Yang, M., and Fu, Q. 2014. "Progress on the Morphological Control of Conductive Network in Conductive Polymer Composites and the Use as Electroactive Multifunctional Materials." Prog. Polym. Sci. 39: 627-55.

[2] Haldorai, Y., Shim, J. J., and Lim, K. T. 2012. "Synthesis of Polymer-inorganic Filler Nanocomposites in Supercritical $\mathrm{CO}_{2}$." J. Supercrit. Fluids 71: 45-63.

[3] Kango, S., Kalia, S., Celli, A., Njuguna, J., Habibi, Y., and Kumar, R. 2013. "Surface Modification of Inorganic Nanoparticles for Development of Organic-inorganic Nanocomposites-A Review." Prog. Polym. Sci. 38: 1232-61.

[4] Adschiri, T. 2007. "Supercritical Hydrothermal Synthesis of Organic-inorganic Hybrid Nanoparticles." Chem. Lett. 36: 1188-93.

[5] Yang, L., Phua, S. L., Toh, C. L., Zhang, L., Ling, H., Chang, M., Zhou, D., Dong, Y., et al.. 2013. "Polydopamine-coated Graphene as Multifunctional Nanofillers in Polyurethane." RSC Adv. 3: 6377.

[6] Yorifuji, D., and Ando, S. 2011. "Enhanced Thermal Conductivity over Percolation Threshold in Polyimide Blend Films Containing ZnO Nano-pyramidal Particles: Advantage of Vertical Double Percolation Structure." J. Mater. Chem. 21: 4402-7.

[7] Chen, Q., Udomsangpetch, C., Shen, S. C., Liu, Y. C., Chen, Z., and Zeng, X. T. 2009. "The Effect of AlOOH Boehmite Nanorods on Mechanical Property of Hybrid
Composite Coatings." Thin Solid Films 517: 4871-4.

[8] Yu, W., Zhao, J., Wang, M., Hu, Y., Chen, L., and Xie, H. 2015. "Thermal Conductivity Enhancement in Thermal Grease Containing Different CuO Structures." Nanoscale Res. Lett. 10: 113.

[9] Nguyen, T. D. 2013. "From Formation Mechanisms to Synthetic Methods Toward Shape-controlled Oxide Nanoparticles." Nanoscale 5: 9455-82.

[10] Taguchi, M., Yamamoto, N., Hojo, D., Takami, S., Adschiri, T., Funazukuri, T., and Naka, T. 2014. "Synthesis of Monocarboxylic Acid-modified $\mathrm{CeO}_{2}$ Nanoparticles Using Supercritical Water." RSC Adv. 4: 49605-13.

[11] Fujii, T., Kawasaki, S-i., Suzuki, A., and Adschiri, T. 2016. "High-Speed Morphology Control of Boehmite Nanoparticles by Supercritical Hydrothermal Treatment with Carboxylic Acids." Cryst. Growth Des. 16: 1996-2001.

[12] Duwe, S., Arlt, C., Aranda, S., Riedel, U., and Ziegmann, G. 2012. "A Detailed Thermal Analysis of Nanocomposites Filled with $\mathrm{SiO}_{2}$, AlN or Boehmite at Varied Contents and a Review of Selected Rules of Mixture." Composites Sci. Tech. 72: 1324-30.

[13] Li, J., Addai-Mensah, J., Thilagam, A., and Gerson, A. R. 2012. "Growth Mechanisms and Kinetics of Gibbsite Crystallization: Experimental and Quantum Chemical Study." Cryst. Growth Des. 12: 3096-103.

[14] Wagner, W., and Pruß, A. 2002, "The IAPWS Formulation 1995 for the Thermodynamic Properties of Ordinary Water Substance for General and Scientific Use." J. Phys. Chem. Ref. Data 31: 387-535.

[15] Lemmon, E. W., Huber, M. L., and McLinden, M. O. 2010. "NIST Standard Reference Database 23: Reference Lluid Thermodynamic and Transport Properties-REFPROP, Version 9.0.” National Institute of Standards and Technology, Standard Reference Data Program, Gaithersburg.

[16] Salvatierra, D., Taylor, J. D., Marrone, P. A., and Tester, J. W. 1999. "Kinetic Study of Hydrolysis of Methylene Chloride from 100 to $500{ }^{\circ} \mathrm{C}$." Ind. Eng. Chem. Res. 38: 4169-74.

[17] Adschiri, T., Hakuta, Y., Sue, K., and Arai, K. 2001. "Hydrothermal Synthesis of Metal Oxide Nanoparticles at Supercritical Conditions." J. Nanopart. Res. 3: 227-235.

[18] Jordan, G., and Rammensee, W. 1996. "Dissolution Rates and Activation Energy for Dissolution of Brucite(001): A New Method Based on the Microtopography of Crystal Surfaces." Geochim. Cosmochim. Acta 60: 5055-62. 